\title{
DISTAL MIGRATION OF THE RODS OF A CONSTRAINED POLYAXIAL PEDICLE SCREW SYSTEM
}

\author{
(1) Tiro MMOPELWA2 , (1) Prashant ADHIKARI², (1) Vugar NABI², (1) Selim AYHAN³, (1) Emre ACAROǦLU4 \\ ${ }^{1}$ Başkent University Hospital, Clinic of Neurological Surgery, Ankara, Turkey \\ ${ }_{2}^{2}$ Acibadem Ankara Hospital ARTES Spine Center, Clinic of Orthopaedics and Traumatology, Ankara, Turkey \\ ${ }^{3}$ Acıbadem Ankara Hospital ARTES Spine Center, Clinic of Neurological Surgery, Ankara, Turkey \\ ${ }^{4}$ Ankara Spine Center, Department of Orthopaedics and Traumatology, Ankara, Turkey
}

\begin{abstract}
Spinal instrumentation and surgical techniques have exponentially improved over the years, and today's spinal surgeon is well equipped to rigidly fix the spine with minimum adverse effects. Complications may emerge during or after the surgical operations. Infection, hematoma and neurological deficits are early noticed findings. Instrumentation problems i.e. screw and/or rod failures present in long-term after surgery. Caudal rod migration out of the spinal column is a rare entity. We report here three case incidents (in two patients) of lumbar degenerative disorders requiring spinal instrumentation that represented with caudal rod migration, all associated with one particular implant made.

Keywords: Implant failure, lumbar spine, migration, pedicle screw instrumentation, spinal stenosis
\end{abstract}

\section{INTRODUCTION}

Spinal instrumentation and surgical techniques have exponentially improved over the years and today's spinal surgeon is well equipped to rigidly fix the spine with minimum adverse effects. But, especially for adult spinal deformity surgery, up to one third of patients experience some form of radiographic and/or implant-related complications, ranging from rod or screw breakage to implant prominence ${ }^{(1)}$. Distant rod migration after spine instrumentation is not a common complication but could potentially result in high morbidity or even fatal outcomes if unrecognized. There are plenty of case reports in the literature about this type of complication following spinal instrumentation. These reports range from cephalic to caudal rod migrations, which in some instances lead to near catastrophes ${ }^{(2-5)}$. There are cases of rod migrating from cervical spine fixation to the occipital fossa, into the brain matter and those of rod migrating from lumbar spine fixation down to the knee ${ }^{(3,5,6)}$. Interestingly, none of all these published reports describe any propensity of this type of complication happening with any particular implant brand, make or metal type. We report here three case incidents (in two patients) of lumbar spinal instrumentation that represented with caudal rod migration, all associated with one particular implant make.

\section{CASE REPORTS}

\section{Case 1}

A 69-year-old male patient presented to our spine clinic with bilateral buttock and lower extremity pain associated with neurogenic claudication. He had no other comorbidities of significance; his body mass index (BMI) value was 34.2. His left extremity pain was worse than the right side. Motor strength was within normal limits and there were no bowel or bladder symptoms. Radiographs and magnetic resonance imaging of the lumbar spine revealed degenerative changes and a flat back with multi-level spinal stenosis. After failure of conservative treatment, he was scheduled for spinal decompression and instrumented fusion. His surgery consisted of posterior decompression through multiple laminectomies as well as facet osteotomies to restore his lordosis and posterior instrumented fusion (using local iliac crest bone) from T12 to sacrum using a constrained polyaxial pedicle screw system with a $6.0 \mathrm{~mm}$ rod diameter (Xia Titanium Spinal System, Stryker Spine, Allendale, $\mathrm{NJ}$, USA) (Figure 1a, b) . Two $6.0 \mathrm{~mm}$ titanium rods and multilevel titanium polyaxial screws were used. Early post-operative period was uneventful, and the patient was discharged home four days after surgery. At three months during patient's routine 
follow-up, lumbar-sacral radiographs revealed a broken left rod now lodged at the gluteal area due to caudal migration (Figure 1c, d). On further questioning, the patient reported some mild aching pain over his left buttock area especially with sitting, but denied any recent strenuous activity. There was no evidence of fusion yet at the instrumented levels. He subsequently underwent a revision surgery of his broken left rod with an additional bypass rod at the left side (Figure 1e, f). Intra-operatively, all the screw caps on the left side were loose. They appeared to be well centered but had backed off by one or two turns allowing the rod to slide down. The screws on the right side were tight. There was no evidence of infection or metallosis. Swabs for cultures were taken and all came back negative for infection.

\section{Case 2}

Twelve months after his second operation, "Case 1" presented to his local medical facility again complaining of left gluteal swelling and redness. According to accompanying notes, there was no evidence for local infection or gluteal abscess. Radiographs taken revealed a left sided distal domino failure and had caudally migrated and lodged at his left gluteal area (Figure 2a, b). Similarly, there was no history of recent trauma or strenuous activity. A third operation was subsequently performed for rod and screws exchange with another spinal instrumentation system (CD Horizon Engage 6.35 Spinal System, Medtronic, Minneapolis, MN USA) (Figure 2c, d). Intra-operative findings were strikingly similar to his previous surgery. Right rod was intact, screw caps on the left sided were well centered but had backed off one or two turns. There was no evidence of
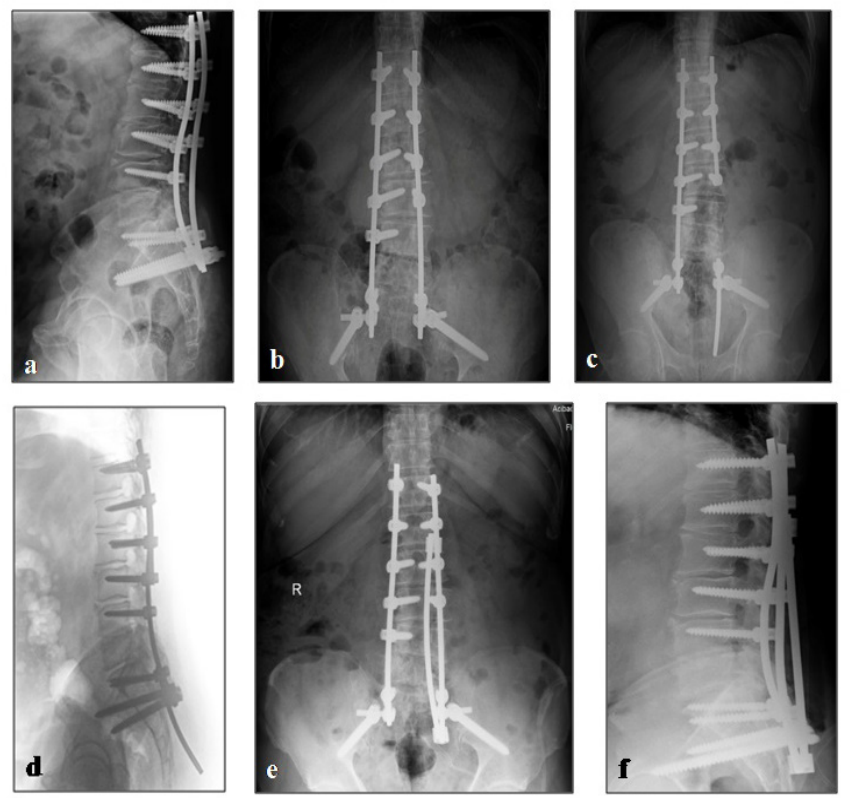

Figure 1. Early post-operative X-ray, AP (a) and lateral view (b), a repeat X-ray at three months AP (c) and lateral (d) views showing a caudally migrated left rod. X-rays after revision surgery, AP (e) and lateral (f) views

AP: Anteroposterior infection or metallosis. He had further follow-up of 14 months with no residual symptoms at the time of the writing of this report.

\section{Case 3}

A 72-year-old male, who had a background history of L1-3 decompression and instrumented fusion for degenerative spinal stenosis performed at another institution 5 years before presenting to our center, was admitted. He had further comorbidities of diabetes mellitus for 32 years, fungal osteomyelitis of the maxilla (treated) and a BMI of 34.6. He presented with the recurrence of low back symptoms and was diagnosed as having further multi-level degenerative spinal stenosis and sagittal imbalance. Similar to the first patient, decompression as well as long instrumented fusion (using local iliac crest bone) from T9 to ileum with partial restoration of lumbar lordosis was performed. Two $6.0 \mathrm{~mm}$ titanium rods and multi-level titanium polyaxial screws of the same instrumentation system as case 1 were used for fixation (Xia Titanium Spinal System, Stryker Spine, Allendale, NJ, USA). His immediate post-operative period was unremarkable. Twelve months later, the patient presented back to our institution complaining of left gluteal pain and swelling. He denied any recent strenuous activity. Further evaluation and radiographs revealed a loose left rod that had migrated caudally to the gluteal area similar to the first patient (Figure 3a,b). The left rod was revised and intra-operative findings revealed loose caps on the left side (seven in total). The caps were well centered but had backed off. There were no signs of infection or metallosis noted and culture swabs were negative. His further follow-
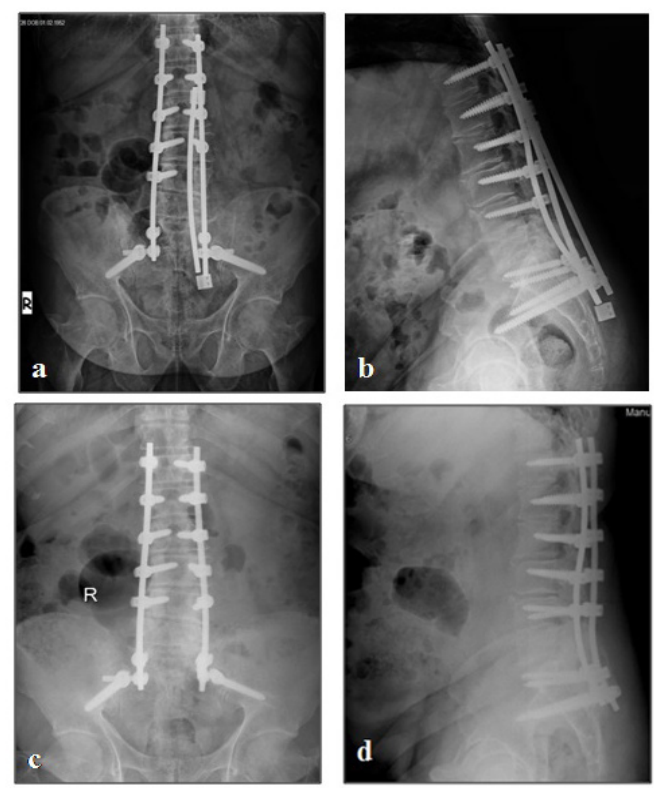

Figure 2. One year after post revision surgery showing caudally migrated domino AP (a) and Lateral (b) X-rays views. Rod and screws exchange with another spinal instrumentation system AP (c) and Lateral (d) X-rays views

AP: Anteroposterior 
up of two years now is uneventful with regard to any further instrumentation problems.

\section{DISCUSSION}

This is a report of two patients who had undergone surgery consisting of decompression and instrumented fusion for lumbar spinal stenosis. These patients experienced rod loosening and migration secondary to the loosening of the screw caps with or without associated rod breakage at a total of three instances. Such a complication associated with very similar patients and a single instrumentation system has not been reported previously.

The case reports of spine rod migrations are mainly of either Harrington rods or of Luque rod instrumentation system, both unconstrained systems in regard to the anchor-rod interface ${ }^{(7)}$. In all these cases described, there was a significant time lapse between the index surgery and discovery of a migrated rod. The three incidents presented here are those of loss of rod fixation and subsequent migration involving one particular implant and all happening within a short span of time from the index surgery. Davne and Myers $^{(8)}$ described $5.6 \%$ of nut loosening in their series of 486 patients and they attributed this as technical problems, this problem was resolved after the introduction of integral-nut screw system. Leute et al.(9) reported a case of set screw fracture with cage dislocation after an open TLIF procedure, they acknowledged this as malpositioning of set screws or flaws in their production. In another case by Bayri et al. (7), a patient, who had underwent spinal instrumentation surgery for spondylolisthesis 6 years ago, was detected to have migration of rod into retroperitoneal region, the reason for movement of the rod in this case was due to unbalanced motion at the instrumented level without any fusion.

This type of implant failure may be secondary to several reasons: technique related, implant design defects, patient related factors and material failure with or without a non-rigid fixation (pseudoarthrosis). Poor technique in the form of insufficient tightening of the nut into the screw head, or improper coupling
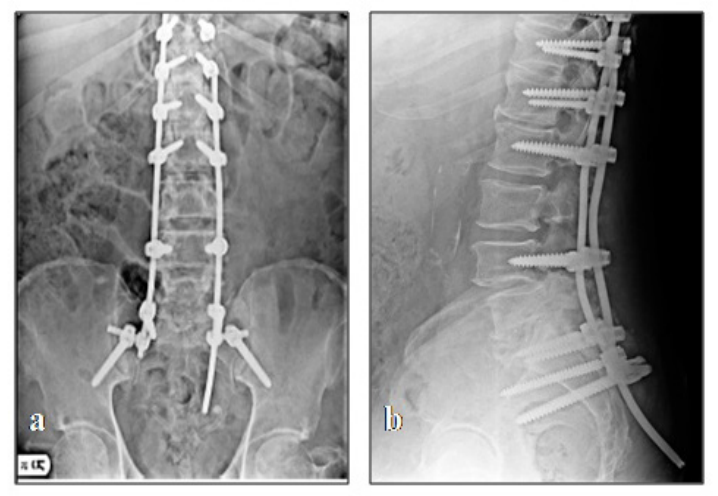

Figure 3. Post-operative X-rays AP (a) and Lateral (b) subsequent sequential failure of fixation with rod migration.

AP: Anteroposterior between nut and screw head can cause loosening and consequent dislodgment of the $\operatorname{rod}^{(10)}$. In our setting, the same team highly experienced in spinal instrumentation operated the two patients in a similar fashion to all other cases. A total of 68 patients were operated with such spinal instrumentation system (Xia Titanium Spinal System, Stryker Spine, Allendale, NJ, USA) upon having had this confusing complication. Screw nuts were tightened in accordance with the recommendations of the manufacturer (a torque wrench screw driver was used with the recommended level of torque application). Hence, surgical technique is highly unlikely to be a factor because this phenomenon has not been experienced when using different implant systems. In addition, there were no signs of eccentric coupling between the nut and screw head intra-operatively at the index surgeries or at the times of revision.

Although it has been reported that these rods can get loose and migrate leaving behind a tight and properly placed pedicle screw caps without evidence of loosening(7), in our cases, the screw caps were loose and backed-off by one or two turns. It is unclear why the caps got loose although after experience with the first case, additional time was spent ensuring that the torque applied to the caps was of sufficient amount as prescribed by the manufacturer. As a second thought, one risk factor that might have been important in regard to manufacturing may be the torque wrench/driver. It is quite plausible that the torque settings of this tool might have been less than accurate resulting in less than ideal tightening of the screw caps. The fact that these failures were noted back to back within a limited time frame ( 2 years before the writing of this report) may be suggestive of such a manufacturing error (of the caps or the torque wrench) in a certain party of implants/instruments.

Metal corrosion and shredding leading to implant loosening are also important aspects to consider especially the interactions between titanium pedicle screw and a different metal such as Cobalt-Chromium ( $\mathrm{CoCr}$ ). This interface is under significant frictional load and can sustain crevice corrosion, metallosis with subsequent loosening ${ }^{(11)}$. In both of our patients described above, we used titanium screws on a titanium rod and there was no evidence of metallosis or corrosive loosening intraoperatively. The magnitude of stress on pedicle screws under impact or dynamic loads depends on the mechanical properties of the rod. Titanium rods are less stiff compared to $\mathrm{CoCr}$ rods and essentially concentrate less stress on the pedicle screws; therefore, biomechanically titanium on titanium is expected to have less stress concentration ${ }^{(4)}$. Other factor to consider is bacteria- induced metal corrosion leading to rod loosening. Propionibacterium acnes have been linked to late infections and implant corrosion and metallosis ${ }^{(12)}$. In the two patients described above, there was no evidence of infection from intraoperative cultures.

Of factors that might have been related to the specific patients, our patients were fairly similar in terms of gender and body composure. BMIs at the range of obesity might have been a contributing factor in this type of failure but most 
probably not as a single decisive factor. Another potential contributing (patient related) factor may be pseudoarthrosis. It is quite plausible that at least one of our patients (case 1) had developed pseudoarthrosis as he had to have another revision surgery for further implant failure. Pseudoarthrosis is expected to impact significant stress on the screw-rod junction in spinal implants but there are no studies in literature directly linking pseudoarthrosis to rod migration. In addition, implant related problems in association with the development of pseudoarthrosis per se would be more likely to result in later (one year and on) failures with a different pattern (rod and/or screw breakage or screw loosening).

It is important to note that rod breakage with subsequent migration can have devastating consequences. Lark et al. ${ }^{(13)}$ have described a case of migrated rod presenting with acute sensory changes in lower extremities with imaging showing rod failure, resulting in the penetration of the rectal wall. AlBinali et al. ${ }^{(2)}$ have reported on a child who presented with a migrated lumbar spinal instrumentation causing massive acute lower gastrointestinal bleeding due to internal iliac artery injury and bowel perforation. This clearly shows that spinal surgeons must have high index of suspicion for any patient presenting with unusual symptoms, on a background history of spinal instrumentation either anterior or posterior.

In summary, we could not identify the reasons for rod loosening and disengagement twice in one patient and another patient, all happening with the same instrumentation system and within a relatively short period of time. Also, the object of interest was that the loss of fixation happened at multiple fixation points rose suspicion of a potential inherent biomechanical weakness with this particular instrumentation system.

To conclude, implant/hardware related problems are rare complications in spine surgery; however, this should be kept in mind and this may lead to a potentially catastrophic condition. Although rare and can happen with any spinal instrumentation system, here, it appears to be higher propensity of disengagement and loosening with previously approved and tested Stryker system (Xia Titanium Spinal System, Stryker Spine, Allendale, NJ, USA). This potential biomechanical problem needs to be further investigated.

\section{Ethics}

Informed Consent: Informed consent was obtained from the patients.

\section{Authorship Contributions}

Surgical and Medical Practices: T.M., V.N.N., S.A., E.A., Concept: T.M., P.A., V.N.N., S.A., E.A., Design: T.M., S.A., E.A., Data
Collection or Processing: T.M., P.A., V.N.N., S.A., E.A., Analysis or Interpretation: T.M., V.N.N., S.A., E.A., Literature Search:T.M., P.A., V.N.N., S.A., E.A., Writing: T.M., P.A., V.N.N., S.A., E.A.

Conflict of Interest: No conflict of interest was declared by the authors.

Financial Disclosure: The authors declared that this study received no financial support.

\section{REFERENCES}

1. Soroceanu A, Diebo BG, Burton D, Smith JS, Deviren V, Shaffrey C, et al; International Spine Study Group. Radiographical and ImplantRelated Complications in Adult Spinal Deformity Surgery: Incidence, Patient Risk Factors, and Impact on Health-Related Quality of Life. Spine (Phila Pa 1976). 2015;40:1414-21.

2. Al-Binali AM, Sigalet D, Goldstein S, Al-Garni A, Robertson M. Acute lower gastrointestinal bleeding as a late complication of spinal instrumentation. J Pediatr Surg. 2001;36:498-500.

3. Dhatt S, Kumar S, Arora N, Dhillon M, Tripathy SK. Migration of anterior spinal rod from the dorsolumbar spine to the knee: an unusual complication of spinal instrumentation. Spine (Phila Pa 1976). 2010;35:E270-2.

4. Doulgeris J. Biomechanical comparison of titanium and cobalt chromium pedicle screw rods in an unstable cadaveric lumbar spine. : Mechanical Engineering, University of South Florida; 2013.

5. Wood KE, Fitch RD, Burton DC, Keiger C). Anterior scoliosis rod migration to the lower extremity. Spine J. 2009;9:e9-12.

6. Yablon IG, Cowan S, Mortara R. The migration of a Harrington rod after cervical fusion. Spine (Phila Pa 1976). 1993;18:356-8.

7. Bayri Y, Eksi MS, Dogrul R, Koc DY, Konya D. Migration of rod into retroperitoneal region: a case report and review of the literature. Korean J Spine. 2014;11:241-4.

8. Davne SH, Myers DL. Complications of lumbar spinal fusion with transpedicular instrumentation. Spine (Phila Pa 1976). 1992;17(6 Suppl):S184-9.

9. Leute PJ, Hammad A, Hoffmann I, Hoppe S, Klinger HM, Lakemeier S. Set screw fracture with cage dislocation after two-level transforaminal lumbar interbody fusion (TLIF): a case report. J Med Case Rep. 2015;9:22.

10. Agrawal A. Pedicle screw nut loosening: potentially avoidable causes of spine instrumentation failure. Asian Spine J. 2014;8:224-6.

11. Yamaguchi K, Konishi $H$, Hara S, Motomura Y. Biocompatibility studies of titanium-based alloy pedicle screw and rod system: histological aspects. Spine J. 2001;1:260-8.

12. Beguiristain I, del Rio J, Duart J, Barroso J, Silva A, Villas C. Corrosion and late infection causing delayed paraparesis after spinal instrumentation. Journal of pediatric orthopedics. J Pediatr Orthop B. 2006;15:320-3.

13. Lark RK, Caputo AM, Brown CR, Michael KW, Thacker JK, Richardson W]. Distant Harrington rod migration 35 years after implantation. J Clin Neurosci. 2013;20:1452-3. 